\title{
Crisis adrenérgica como forma de debut de un neuroblastoma
}

\author{
Adrenergic crisis as a debut form of a neuroblastoma \\ José Antonio Alonso Cadenas ${ }^{\mathrm{a}}$, José Luis Almodóvar Martín ${ }^{\mathrm{b}}$, María Isabel Iglesias Bouzas ${ }^{\mathrm{b}}$, \\ Raquel Jiménez García ${ }^{c}$, Ana Serrano González
}

\author{
aServicio de Urgencias. Hospital Infantil Universitario Niño Jesús. Madrid, España \\ bServicio de Cuidados Intensivos. Hospital Infantil Universitario Niño Jesús. Madrid, España \\ cServicio de Pediatría. Hospital Infantil Universitario Niño Jesús. Madrid, España
}

Recibido: 20 de febrero de 2020; aceptado: 17 de mayo de 2020

\section{¿Qué se sabe del tema que trata este estudio?}

El neuroblastoma es el tumor sólido extracraneal más frecuente en pediatría y puede originarse a lo largo de toda la cadena neuronal simpática. Su forma clínica más frecuente de presentación es la abdominal en forma de dolor o distensión.

\section{¿Qué aporta este estudio a lo ya conocido?}

Se describe una forma atípica de presentación del neuroblastoma con hipertensión arterial, taquicardia, sudoración e irritabilidad en forma de crisis adrenérgica por liberación de catecolaminas. El diagnóstico diferencial es variado y debe realizarse con patología cardiaca, metabólica (hipoglucemia) y/o intoxicaciones.

\section{Resumen}

La forma clínica de presentación más común del neuroblastoma es el de una masa abdominal, pero puede presentarse con sintomatología menos habitual, como es la crisis adrenérgica por liberación de catecolaminas. Objetivo: Describir una forma de presentación inusual de neuroblastoma y el amplio diagnóstico diferencial que existe en un lactante con síntomas adrenérgicos. Caso Clínico: Lactante femenina de 7 semanas de vida, consultó por historia de tres semanas de sudoración e irritabilidad a lo que se asoció fiebre de $24 \mathrm{~h}$ de evolución y dificultad respiratoria. Al ingreso presentaba mal estado general, irritabilidad, sudoración, enrojecimiento facial, taquipnea y palidez cutánea, taquicardia sinusal extrema e hipertensión arterial (HTA), interpretadas como sintomatología adrenérgica. Se completó el estudio con una ecografía abdominal y resonancia magnética que mostraron una gran masa retroperitoneal compatible con neuroblastoma. Las catecolaminas en sangre y en orina mostraron altos niveles de dopamina, adrenalina y noradrenalina, probablemente de origen tumoral. Se inició tratamiento antihipertensivo con fármacos alfa bloqueantes con buen control de la tensión arterial. Se resecó quirúrgicamente el tumor sin incidencias y con una adecuada recuperación posterior. La paciente presentó evolución favorable a tres años de seguimiento. Conclusiones: en un lactante con sintomatología adrenérgica como irritabilidad, enrojecimiento, sudoración asociada a HTA, se debe descartar patología cardiaca, metabólica (hipoglucemia), intoxicaciones y/o patología suprarrenal. Dentro de esta última, el neuroblastoma es la primera posibilidad diagnóstica, por ser uno de los principales tumores en la infancia y aunque esta presentación no es habitual puede producir estos síntomas.

Correspondencia:

José Antonio Alonso Cadenas

jalonsocadenas@gmail.com 


\begin{abstract}
The most common clinical presentation of neuroblastoma is an abdominal mass, but it can present with uncommon symptoms, such as adrenergic storm due to catecholamine release. Objective: To describe an unusual presentation of neuroblastoma and the wide differential diagnosis that exists in an infant with adrenergic symptoms. Clinical Case: A 7-week old female infant was evaluated due to a 3-week history of sweating and irritability associated with a 24-hour fever and respiratory distress. At admission, she presented poor general condition, irritability, sweating, facial redness, tachypnea and skin paleness, extreme sinus tachycardia, and high blood pressure (HBP), interpreted as adrenergic symptoms. The study was completed with abdominal ultrasound and magnetic resonance imaging that showed a large retroperitoneal mass compatible with neuroblastoma. Plasma and urinary catecholamines tests showed high levels of dopamine, adrenaline, and noradrenaline, probably of tumor origin. We started antihypertensive treatment with alpha-blocker drugs, showing a good blood pressure control. The tumor was surgically resected without incidents and adequate subsequent recovery. The patient presented a favorable evolution after three years of follow-up. Conclusions: In an infant with adrenergic symptoms such as irritability, redness, sweating associated with HBP, it should be ruled out pathology heart or metabolic (hypoglycemia) pathology, intoxications, and/or adrenal pathology. Within this last one, neuroblastoma is the first diagnostic possibility, since it is one of the main tumors in childhood and, although this presentation is not usual, it can produce these symptoms.
\end{abstract}

\section{Keywords:}

Nephrology;

Arterial Hypertension;

Oncology;

Neoplasms;

Neuroblastoma

\section{Introducción}

El neuroblastoma es el tumor sólido extracraneal más frecuente en pediatría y comprende un $6-8 \%$ de todos los cánceres pediátricos ${ }^{1-6}$. Puede originarse a lo largo de toda la cadena neuronal simpática ${ }^{1-4}$. La relación varón-mujer es 1,2:1 1,2. La edad media de los pacientes en el momento del diagnóstico es de 2 años, detectándose el $90 \%$ de los casos en menores de 5 años ${ }^{1-3}$. La etiología es desconocida y hasta la fecha no ha sido identificado ningún factor ambiental pre o postnatal desencadenante ${ }^{3}$. La mayoría de los casos son esporádicos, pero existe un 1-2\% de ellos con historia familiar previa $^{3,4}$.

En general se presenta de manera aislada, pero puede asociarse a otros síndromes como la enfermedad de Hirschprung, el síndrome de Ondine, la enfermedad de von Recklinghausen y la hipomelanosis de Ito ${ }^{4}$. Se caracteriza por tener un comportamiento muy heterogéneo, que varía desde tumores de diagnóstico casual en la infancia con una supervivencia de más del 90\%, mejor pronóstico en los menores de un año, en contraste con aquellos que tienen un comportamiento altamente agresivo, debutan con metástasis y presen$\tan$ una baja supervivencia ${ }^{1-6}$. Se han encontrado tumores suprarrenales durante las autopsias de fetos y niños prematuros ${ }^{3,4}$. Por alguna razón el tumor regresa espontáneamente en la mayoría de los sobrevivientes, haciéndose clínicamente inapreciable mientras que, en otros casos, se diferencian hacia tumores benignos más adelante, una vez que han nacido ${ }^{1,3}$.

Los síntomas de presentación suelen ser bastante inespecíficos y pueden ser causados por el tumor primario, por metástasis o por alteraciones metabólicas ${ }^{1-6}$. El tumor primario se encuentra en el abdomen en el $60 \%$ de los casos y en más de la mitad de las ocasiones se sitúa en la glándula suprarrenal ${ }^{1-6}$. La segunda localización en frecuencia es en el tórax que representa un $14 \%$ de todos los casos, seguido de pelvis, $6 \%$, y región cervical, $2 \%{ }^{1-6}$. Hasta en un $18 \%$ está en otras localizaciones distintas ${ }^{1-6}$. La forma clínica de presentación más frecuente de los tumores abdominales es como masa abdominal que puede provocar dolor abdominal, distensión o, raramente, obstrucción intestinal ${ }^{1-3}$. Los tumores torácicos pueden ocasionar tos, disfagia, dificultad respiratoria, síndrome de la vena cava superior o síndrome de Horner ${ }^{1-3}$. Este último también puede ser producido por los tumores de la región cervical. Los tumores de localización pélvica pueden causar obstrucción urinaria o estreñimiento ${ }^{1-3}$. Los lugares más frecuentes de metástasis son ganglios linfáticos, hígado, piel, huesos y médula ósea ${ }^{1-3,5}$. Pueden tener sintomatología diversa como dolor óseo, cojera, compresión medular, equimosis periorbitaria y proptosis que son denominados como "ojos de mapache" y pueden presentar en la exploración física una hepatomegalia de crecimiento rápido ${ }^{1-3,5}$. Además pueden asociar distintos síndrome paraneoplásicos como el síndrome opsoclono-mioclono que cursa con ataxia, mioclonías y movimientos erráticos de los ojos; diarrea secretora intensa, rubor, sudoración e hipertensión arterial (HTA) por aumento en la producción de péptido intestinal vasoactivo, o, más raramente, en forma de HTA, taquicardia y sudoración por liberación de cate- 
colaminas ${ }^{1-3,5}$. En esta última situación, la irritabilidad también puede estar presente y ser el equivalente de la cefalea en el niño mayor como parte de la sintomatología adrenérgica ${ }^{1-3,5}$.

Los signos y síntomas de sobreactividad simpática producidos por una elevación en las resistencias vasculares, aumento del consumo de oxígeno y un estado hiperdinámico con aumento de la postcarga son muy inusuales y pueden pasar desapercibidos provocando la evolución de estos pacientes a situaciones de insuficiencia cardiaca, shock e incluso ocasionar la muerte ${ }^{2,4}$. Es de vital importancia identificar a estos pacientes de manera precoz para evitar su descompensación metabólica, la diseminación del tumor a otros órganos y así mejorar su pronóstico.

El objetivo del presente manuscrito es describir una forma de presentación inusual de neuroblastoma y el amplio diagnóstico diferencial que existe en un lactante con síntomas adrenérgicos.

\section{Caso Clínico}

Lactante femenina de un mes y tres semanas de vida que es llevada al servicio de urgencias por fiebre y dificultad respiratoria de 24 h de evolución. Los padres refirieron que aproximadamente tres semanas antes notaron a la niña más irritable y con sudoración de manera casi constante, sin ningún desencadenante evidente. Su alimentación era con fórmula adaptada de inicio, sin cambios desde el nacimiento, con adecuado incremento ponderal. Tenía antecedentes de haber nacido a término con peso adecuado para la edad gestacional $(3.910 \mathrm{~g})$. Embarazo y parto sin complicaciones. Sus pruebas metabólicas (TSH y PKU) fueron normales. Padres no consanguíneos. Padre con antecedente de coartación de aorta intervenida en periodo neonatal. Sin otros antecedentes de relevancia.

En la evaluación inicial destacaba regular estado general, por presentar dificultad respiratoria con marcado tiraje sub e intercostal, irritabilidad y mala perfusión distal. La saturación inicial era 97\%, frecuencia respiratoria $68 \mathrm{rpm}$, frecuencia cardiaca $231 \mathrm{lpm}$, tensión arterial (TA) 119/84 mmHg, glucemia $112 \mathrm{mg} /$ dl y temperatura axilar $39,5{ }^{\circ} \mathrm{C}$. La vía aérea estaba permeable y la auscultación pulmonar era normal. En la auscultación cardiaca destacaba taquicardia mantenida con ritmo de galope. Los pulsos eran palpables en las cuatro extremidades y el relleno capilar estaba enlentecido (3 segundos) con frialdad a nivel distal en extremidades inferiores. Pupilas normorreactivas e isocóricas. Enrojecimiento facial sin lesiones en la piel. Al examen, el abdomen era blando y depresible sin aparentes masas ni visceromegalias. Resto del examen sin alteraciones. Tenía movimientos simétricos y espontáneos en las cuatro extremidades, buen tono muscular y sin focalidad neurológica.

Se inició oxigenoterapia en mascarilla con reservorio. Se canalizaron dos vías venosas periféricas para extracción de exámenes de sangre (tabla 1) y se infundieron $10 \mathrm{ml} / \mathrm{kg}$ de suero salino fisiológico. Se administró antitérmico y una dosis de antibiótico de amplio espectro i.v.

El electrocardiograma objetivó una taquicardia sinusal extrema con una frecuencia de $225 \mathrm{lpm}$ y una ecocardiografía con un corazón estructural y funcionalmente normal que descartó patología cardiaca. Análisis de sedimento y tóxicos en orina sin alteraciones.

Durante su estancia en el servicio de urgencia, su frecuencia cardiaca y su TA continuaron por encima del percentil 99 para su edad y la dificultad respiratoria que presentaba desde el inicio no mejoró a pesar de las medidas de estabilización inicial. Ingresó en la unidad de cuidados intensivos pediátricos (UCIP) para monitorización estrecha y completar su estudio.

En la UCIP, la paciente presentó unas cifras de TA compatibles con una crisis hipertensiva. Se consideró la taquicardia, sudoración, HTA y la vasoconstricción periférica como sintomatología adrenérgica; se completó el estudio con una ecografía abdominal para descartar la existencia de un tumor secretor de catecolaminas, además de valorar la repercusión renal y descartar una alteración a ese nivel.

En la ecografía abdominal (figura 1) se objetivó una gran masa retroperitoneal de localización perivertebral derecha con bordes bien definidos que medía aproximadamente $4 \times 4,3 \times 5,4 \mathrm{~cm}$, compatible con neuroblastoma, que se confirmó mediante resonancia

Tabla 1. Exámenes de sangre realizados en la evaluación inicial

\begin{tabular}{lll}
\hline Valores analíticos & Resultados & Valores normales \\
\hline Leucocitos & $14.280 / \mathrm{mm}^{3}$ & $4.000-11.000$ \\
Neutrófilos & $6.420 / \mathrm{mm}^{3}$ & \\
Linfocitos & $7.190 / \mathrm{mm}^{3}$ & \\
Hemoglobina & $10,1 \mathrm{~g} / \mathrm{dl}$ & $10,4-12,5 \mathrm{~g} / \mathrm{dl}$ \\
Plaquetas & $685.000 / \mathrm{mm}^{3}$ & $150.000-400.000 / \mathrm{mm}^{3}$ \\
Glucosa & $94 \mathrm{mg} / \mathrm{dl}$ & $64-100 \mathrm{mg} / \mathrm{dl}$ \\
Urea & $38,4 \mathrm{mg} / \mathrm{dl}$ & $8-35 \mathrm{mg} / \mathrm{dl}$ \\
Creatinina & $0,34 \mathrm{mg} / \mathrm{dl}$ & $0,24-0,42 \mathrm{mg} / \mathrm{dl}$ \\
Sodio & $143 \mathrm{mEq} / \mathrm{l}$ & $134-145 \mathrm{mEq} / \mathrm{l}$ \\
Potasio & $4,8 \mathrm{mEq} / \mathrm{l}$ & $4,3-6,2 \mathrm{mEq} / \mathrm{l}$ \\
Cloro & $108 \mathrm{mEq} / \mathrm{l}$ & $95-111 \mathrm{mEq} / \mathrm{l}$ \\
Proteína C reactiva & $0,57 \mathrm{mg} / \mathrm{dl}$ & $0,01-1 \mathrm{mg} / \mathrm{dl}$ \\
Procalcitonina & $0,11 \mathrm{ng} / \mathrm{ml}^{2}$ & $0,1-0,5 \mathrm{ng} / \mathrm{ml}$ \\
\hline
\end{tabular}


Figura 1. Ecografía abdominal, corte transversal. Gran masa sólida retroperitoneal paravertebral, discretamente lateralizada a derecha de bordes bien definidos (flecha). La lesión es sólida, levemente heterogénea con imágenes hiperecogénicas puntiformes dispersas (calcificaciones).
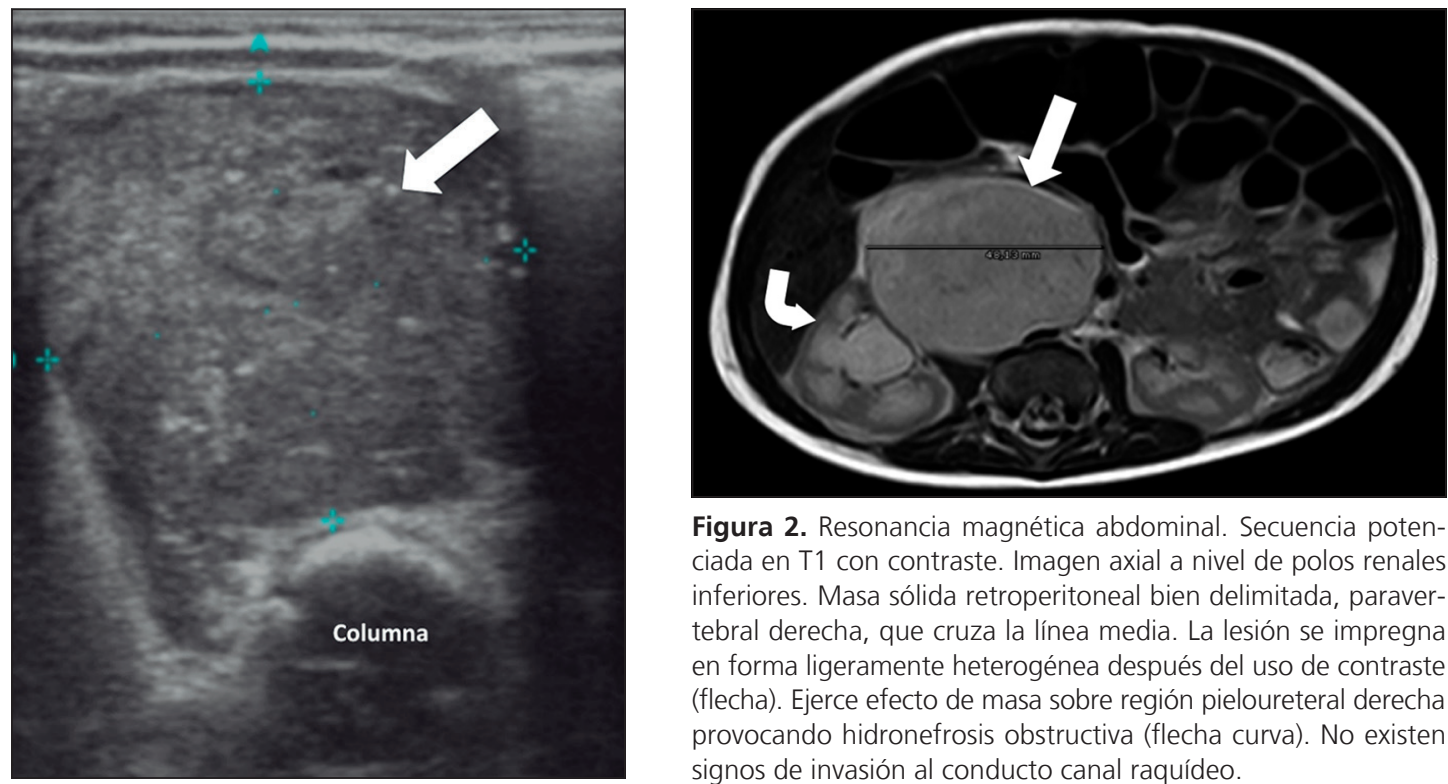

Figura 2. Resonancia magnética abdominal. Secuencia potenciada en T1 con contraste. Imagen axial a nivel de polos renales inferiores. Masa sólida retroperitoneal bien delimitada, paravertebral derecha, que cruza la línea media. La lesión se impregna en forma ligeramente heterogénea después del uso de contraste (flecha). Ejerce efecto de masa sobre región pieloureteral derecha provocando hidronefrosis obstructiva (flecha curva). No existen signos de invasión al conducto canal raquídeo. magnética (figura 2). Posteriormente se realizó una cuantificación de catecolaminas en sangre y en orina (tabla 2) cuyo resultado mostró altos niveles de dopamina, adrenalina y noradrenalina, probablemente de origen tumoral. El estudio de extensión con aspiración y biopsia de médula ósea y radiografía de tórax fue negativo. La gammagrafía con metayodobencilguanidina mostró actividad metabólica en la región retroperitoneal sin lesiones a distancia.

Tabla 2. Catecolaminas en sangre y orina

\begin{tabular}{lll}
\hline Catecolaminas en orina & Valores del paciente & Valores de referencia \\
\hline Adrenalina & $6,12 \mathrm{mcg} / 24 \mathrm{~h}$ & $0-3,5 \mathrm{mcg} / 24 \mathrm{~h}$ \\
Noradrenalina & $18,36 \mathrm{mcg} / 24 \mathrm{~h}$ & $0-15 \mathrm{mcg} / 24 \mathrm{~h}$ \\
Dopamina & $139,46 \mathrm{mcg} / 24 \mathrm{~h}$ & $3-85 \mathrm{mcg} / 24 \mathrm{~h}$ \\
Ácido vanilmandélico & $4,8 \mathrm{mg} / 24 \mathrm{~h}$ & $1-2,6 \mathrm{mg} / 24 \mathrm{~h}$ \\
Ácido homovanílico & $13,24 \mathrm{mg} / 24 \mathrm{~h}$ & $0-4,3 \mathrm{mg} / 24 \mathrm{~h}$ \\
\hline
\end{tabular}

Se inició tratamiento antihipertensivo oral con amlodipino junto con un fármaco alfa bloqueante, fenoxibenzamina, que progresivamente consiguieron disminuir la TA hasta cifras en torno al percentil 90 para la edad de la paciente (TA $90 / 50 \mathrm{mmHg}$ ) y controlar los episodios de crisis adrenérgicas que cada vez presentaban menor repercusión hemodinámica (tabla 3) hasta su completa desaparición el día +18 de ingreso. Previo a la cirugía no se realizó bloqueo betaadrenérgico dado la edad de la paciente, la estabilidad clínica que presentaba y la ausencia de taquicardia refleja.

En el día +25 fue intervenida quirúrgicamente sin incidencias. Se realizó una resección macroscópica del más del 90\% de la tumoración. En el análisis anatomopatológico macroscópico presentaba una coloración parduzca, con áreas de necrosis y calcificación, presentando un aspecto global heterogéneo y en el análisis microscópico se observaban escasos signos de diferenciación hacia célula ganglionar cuyo grupo pronóstico es favorable. A las $24 \mathrm{~h}$ de la intervención pudo suspen-

Tabla 3. Episodios de crisis adrenérgicas durante el ingreso antes de la cirugía

\begin{tabular}{lccc}
\hline Días desde su atención inicial (tratamiento instaurado) & Fc máxima & TA máxima & Sintomatología adrenérgica \\
\hline 0 & 231 & $149 / 104$ & ++++ \\
+1 (fenoxibenzamina cada $24 \mathrm{~h}$ ) & 218 & $126 / 81$ & ++++ \\
+4 (fenoxibenzamina cada $12 \mathrm{~h}$ ) & 170 & $110 / 70$ & +++ \\
+6 (fenoxibenzamina cada $6 \mathrm{~h}$ ) & 206 & $102 / 53$ & ++ \\
+10 (fenoxibenzamina cada $6 \mathrm{~h}$ ) & 158 & - \\
\hline
\end{tabular}

Fc: frecuencia cardiaca; TA: tensión arterial; ++++: grave; +++: moderada; ++: leve; -: ausencia de síntomas. 
derse la sedación y se extubó sin incidencias con una adecuada recuperación.

Evolucionó favorablemente en el postoperatorio, en la evaluación oncológica se decidió actitud expectante por tratarse de un neuroblastoma localizado con histología favorable, resección casi completa y sin amplificación del oncogén N-Myc. Tras el alta, fue seguida durante los siguientes tres años en forma ambulatoria con muy buena evolución.

\section{Discusión}

Frente a una paciente con taquicardia, HTA, sudoración e hipoperfusión periférica se debe considerar una crisis adrenérgica producida por la liberación aumentada de catecolaminas que estimulan los receptores alfa y betaadrenérgicos ${ }^{7}$. Estos síntomas pueden estar producidos por diferentes entidades diagnósticas.

La sudoración tan intensa y la vasoconstricción periférica que presentaba la paciente, podían ser signos de insuficiencia cardiaca secundaria a una cardiopatía congénita no diagnosticada durante la gestación, como una coartación de aorta ${ }^{8}$. La palpación de todos los pulsos y la medición de la TA en las cuatro extremidades resultan imprescindibles para su diagnóstico ${ }^{8}$. El ecocardiograma es la prueba complementaria indicada cuando existe la sospecha ${ }^{8}$. El padre de esta paciente había presentado una coartación de aorta en la infancia, y se conoce que el riesgo de tener un niño con una cardiopatía congénita aumenta cuando uno de sus progenitores la ha tenido?

Dado que la frecuencia cardiaca de la paciente era extremadamente elevada, había que descartar una taquiarritmia, mediante un electrocardiograma con todas las derivaciones. La taquicardia paroxística supraventricular es la taquiarritmia más frecuente en pediatría $^{10}$. Presenta una frecuencia cardiaca regular de 150-300 latidos por minuto y las ondas $\mathrm{P}$ no siempre están presentes ${ }^{10}$.

Otra condición patológica para descartar es la hipoglucemia por ser uno de los trastornos metabólicos más frecuente en pediatría, y su diagnóstico y tratamiento precoz son esenciales para la prevención de secuelas neurológicas ${ }^{11}$. La sintomatología está provocada tanto por los efectos directos de la disminución del aporte energético al sistema nervioso central como por la respuesta adrenérgica ${ }^{11}$. Los síntomas son inespecíficos, describiéndose letargia, apatía, apnea, llanto débil, temblor, irritabilidad, convulsiones, coma, sudoración, taquicardia, vómitos y palidez ${ }^{11}$. En los neonatos y lactantes pequeños la mayoría de las causas son transitorias, por una producción disminuida o una utilización aumentada de la glucosa ${ }^{11}$, pero existen otras que producen hipoglucemia persistente como hiperinsulinismo, hipopituitarismo o enfermedades metabólicas ${ }^{11}$.

También, es importante realizar un despistaje de drogas de abuso en orina como por ejemplo anfetaminas o cocaína que producen HTA, taquicardia y sudoración $^{12}$.

En la monitorización inicial de esta paciente se constataron cifras muy elevadas de TA compatibles con una crisis hipertensiva ${ }^{13}$. Se realizó una ecografía abdominal con estudio Doppler para evaluar la repercusión renal y descartar una alteración en el flujo renal o una estenosis de las arterias renales, encontrando una masa suprarrenal que explicaba la sintomatología adrenérgica. Los principales tumores derivados de la cresta neural son: feocromocitoma, paraganglioma y neuroblastoma ${ }^{14,15}$. Dentro de estos, el feocromocitoma y el paraganglioma se presentan de manera similar a este paciente, pero son muy infrecuentes en el periodo neonatal y en los primeros años de vida ${ }^{14,15}$, mientras el neuroblastoma, que es el tumor sólido extracraneal más frecuente en la infancia $^{1-6,14,15}$, no produce habitualmente sintomatología en relación con la liberación de catecolaminas ${ }^{1-6,17-19}$. No es una causa frecuente de HTA y cuando ocurre suele ser secundaria a compresión renal por la masa tumoral en la mayoría de las ocasiones y no por liberación de catecolaminas ${ }^{16-19}$. Como ocurrió en este caso, los neuroblastomas productores de catecolaminas y especialmente los de predominio excretor dopaminérgico pueden producir enrojecimiento facial, taquicardia e HTA ${ }^{1-3,5}$.

Los tumores secretores de catecolaminas precisan un control específico de la sintomatología adrenérgica, ya que, la excesiva liberación de catecolaminas puede provocar crisis hipertensivas ${ }^{16-21}$. La preparación farmacológica preoperatoria está indicada para los pacientes con neoplasias secretoras de catecolaminas, incluidos los asintomáticos y normotensos ${ }^{16,20,21}$. El bloqueo $\alpha$-adrenérgico debe iniciarse entre 7 y 10 días antes de la cirugía para normalizar la TA, al mismo tiempo que se intenta expandir la volemia mediante un adecuado ajuste de la fluidoterapia y/o transfusión de concentrado de hematíes si fuese necesario ${ }^{16,20,21}$. La fenoxibenzamina es el fármaco más empleado en la preparación preoperatoria para controlar la HTA y las arritmias. Tras conseguir un bloqueo $\alpha$-adrenérgico adecuado, se inicia el bloqueo $\beta$-adrenérgico habitualmente 2 o 3 días antes de la cirugía ${ }^{16,20,21}$. El tratamiento con $\beta$-bloqueantes no debería iniciarse antes, puesto que el efecto de bloqueo sobre los receptores $\beta_{2}$, cuyo estímulo produce vasodilatación periférica, podría dar lugar a mayor vasoconstricción arterial y provocar HTA grave o descompensación cardiopulmonar ${ }^{16,20,21}$. Otros fármacos empleados en el control de la HTA asociada a la descarga de catecolaminas son los anta- 
gonistas del calcio ${ }^{19-21}$, que bloquean el transporte de calcio mediado por noradrenalina al músculo liso vascular. La principal función de estos fármacos es complementar el protocolo de bloqueo combinado alfa y betaadrenérgico cuando el control de la HTA es inadecuado, o bien reemplazarlo, en pacientes con efectos secundarios graves ${ }^{20-22}$.

\section{Conclusiones}

En lactantes que presenten sintomatología adrenérgica como irritabilidad, enrojecimiento, sudoración asociado a HTA, existe un variado diagnóstico diferencial como patología cardiaca, metabólica (hipoglucemia), intoxicaciones y/o patología suprarrenal. Dentro de esta última, el neuroblastoma suele ser uno de los principales tumores en la infancia y aunque esta presentación no es la más habitual, puede producir estos síntomas cuando tienen una liberación aumentada de catecolaminas.

\section{Responsabilidades Éticas}

Protección de personas y animales: Los autores declaran que los procedimientos seguidos se conformaron a las normas éticas del comité de experimentación humana responsable y de acuerdo con la Asociación Médica Mundial y la Declaración de Helsinki.

Confidencialidad de los datos: Los autores declaran que han seguido los protocolos de su centro de trabajo sobre la publicación de datos de pacientes.

Derecho a la privacidad y consentimiento informado: Los autores han obtenido el consentimiento informado de los pacientes y/o sujetos referidos en el artículo. Este documento obra en poder del autor de correspondencia.

\section{Conflicto de intereses}

Los autores declaran no tener conflicto de intereses.

\section{Referencias}

1. Moreno Martín-Retortillo L Neuroblastoma. En: Madero L, Lassaletta A, Sevilla J. ed. Hematología y Oncología Pediátricas: Ergón; 2015;621-31.

2. Maris JM, Hogarty MD, Bagatell R, Cohn SL. Neuroblastoma. Lancet. 2007;369:2016-20.

3. Brodeur G, Hogarty M, Bagatell R, et al Neuroblastoma. In: Pizzo P, Poplack D, eds. Principles and practice of pediatric oncology. Philidelphia, PA: Lippincott Williams \& Wilkins. 2016:772.

4. Irwin MS, Park JR. Neuroblastoma: Paradigm for precision medicine. Pediatr Clin North Am. 2015;62:225-56.

5. Monclair T, Brodeur GM, Ambros PF, et al. The International Neuroblastoma Risk Group (INRG) staging system: an INRG Task Force report. J Clin Oncol. 2009;27:298-303.

6. Nakagawara A, Li Y, Izumi H, Muramori K, Inada H, Nishi M. Neuroblastoma. Jpn J Clin Oncol. 2018;48(3):214-41.

7. Martos-Moreno GA, Pozo-Román J, Argente J. Tumores suprarrenales en la infancia. An Pediatr. 2013;79(3):187.e1187.e16.

8. Gausche-Hill M, Buitenhuys C.: Shock. En: Fuchs S, Yamamoto L. ed. Advanced Life Support Provider Manual: Jones\&Bartlett learning; 2016;96-129.

9. Barriales-Villa R, Gimeno-Blanes JR, Zorio-Grima E, et al. Plan of Action for Inherited Cardiovascular Diseases:
Synthesis of Recommendations and Action Algorithms. Rev Esp Cardiol. 2016;69:300-9.

10. Maconochie IK, Bingham R, Eich C, et al. European Resuscitation Council Guidelines for Resuscitation 2015 Section 6. Paediatric life support. Resuscitation. 2015;95:223-48.

11. Martos-Moreno GA. Hipoglucemia. En: Argente Oliver J, Soriano Guillén L. ed. Manual de Endocrinología Pediátrica: Ergón; 2014;221-31.

12. García-Algar O, Papaseit E, Velasco M, et al. Consulta en urgencias de pediatría por intoxicación aguda por drogas de abuso. An Pediatr. 2011;74:413.e1-413.e9.

13. Flynn JT, Kaelber DC, BakerSmith CM, et al.; subcommittee on screening and management of high blood pressure in children. Clinical Practice Guideline for Screening and Management of High Blood Pressure in Children and Adolescents. Pediatrics. 2017;140(3):e20171904.

14. Ribeiro RC, Pinto EM, Zambetti GP Rodríguez-Galindo C. The International Pediatric Adrenocortical Tumor Registry initiative: Contributions to clinical, biological, and treatment advances in pediatric adrenocortical tumors. Mol Cell Endocrinol. 2012;351:37-43.

15. Ciftci AO, Senocak ME, Tanyel FC, Büyükpamukçu N. Adrenocortical tumors in children. J Pediatr Surg. 2001;36:54954.

16. Madre C, Orbach D, Baudouin V, et al. Hypertension in childhood cancer. A frequent complication of certain tumor sites. J Pediatr Hematol Oncol. 2006;28:659-64.

17. Hernández MR, Shamberger RC, Seefelder C. Catecholamine-secreting neuroblastoma in a 4-month-old infant: perioperative management. J Clin Anesth. 2009;21:54-6

18. Pappas L, Shamberger RC, Seefelder C. Giant, Dopamine Secreting Thoracoabdominal Neuroblastoma in a 2-year-old: Rapid Preoperative Blockade With Labetalol. J Pediatr Hematol Oncol. 2010;32:163-6.

19. Sagarzi M, Smith ML, Worth RC, Roberts NB. A rare ganglioneuroblastoma secreting dopamine and the value of its measurement in diagnosis and prognosis. Ann Clin Biochem. 2006;43:73-6.

20. Neumann HPH, Young WF, Eng C. Pheochromocytoma and Paranganglioma. N Eng J Med. 2019;381(6):552-65.

21. Pacak K, Eisenhofer G, Ahlman H, et al. Pheochromocytoma: recommendations for clinical practice from the First International Symposium. Nature Clinical Practice Endocrinology \& Metabolism. 2007;3(2):92-102.

22. Proye $\mathrm{C}$, Thevenin $\mathrm{D}$, Cecat $\mathrm{P}$, et al. Exclusive use of calcium channel blockers in preoperative and intraoperative control of pheochromocytomas: hemodynamics and free catecholamine assays in ten consecutive patients. Surgery. 1989;106(6):1149-54. 